\title{
Low momentum shell model effective interactions with all-order core polarizations
}

\author{
Jason D. Holt ${ }^{1}$, Jeremy W. Holt ${ }^{1}$, T. T. S. Kuo ${ }^{1}$, G. E. Brown ${ }^{1}$, and S. K. Bogner ${ }^{2}$ \\ ${ }^{1}$ Department of Physics, \\ State Univeristy of New York at Stony Brook \\ Stony Brook, New York 11794, USA \\ ${ }^{2}$ Department of Physics, The Ohio State University, \\ Columbus, Ohio 43210, USA
}

(Dated: July 12, 2018)

\begin{abstract}
An all-order summation of core polarization diagrams using the low-momentum nucleon-nucleon interaction $V_{\text {low-k }}$ is presented. The summation is based on the Kirson-Babu-Brown (KBB) induced interaction approach in which the vertex functions are obtained self consistently by solving a set of non-linear coupled equations. It is found that the solution of these equations is simplified by using $V_{\text {low-k }}$, which is energy independent, and by employing Green functions in the particle-particle and particle-hole channels. We have applied this approach to the $s d$-shell effective interactions and find that the results calculated to all orders using the KBB summation technique are remarkably similar to those of second-order perturbation theory, average differences being less than $10 \%$.
\end{abstract}

PACS numbers: 21.60.Cs, 21.30.-x,21.10.-k

Introduction.- Since the early works of Bertsch [1] and Kuo and Brown 2], the effect of core polarization in nuclear physics has received much attention. Core polarization is particularly important in the shell model effective interactions, where this process provides the long-range inter-nucleon interaction mediated by excitations of the core [3]. In microscopic calculations of effective interactions, core polarization has played an essential role, as illustrated by the familiar situation in ${ }^{18} \mathrm{O}$. There the spectrum calculated with the bare $G$-matrix was too compressed compared with experiment, while the inclusion of core polarization had the desirable effect of both lowering the $0^{+}$ground state and raising the $4^{+}$state, leading to a much improved agreement with experiment [1, 2]. As pointed out by Zuker [4, the Kuo-Brown matrix elements, although developed quite some time ago, continue to be a highly useful shell model effective interaction. It should be noted that the core polarization $(\mathrm{CP})$ diagrams associated with the above interactions were all calculated to second order (in the $G$-matrix) in perturbation theory. But what are the effects of core polarization beyond second order, and how can they be calculated? In this Letter we would like to address these questions and present an all-order summation of CP diagrams for the $s d$-shell interactions.

There have been a number of important CP studies beyond second order. Third-order core polarization diagrams, including those with one fold, were studied in detail by Barrett and Kirson [5] for the $s d$-shell effective interactions. Horth-Jensen et al. [6] have carried out extensive investigations of the third-order CP diagrams for the tin region. A main result of these studies is that the effect of the third-order diagrams is generally comparable to that of the second order; the former cannot be ignored in comparison with the latter. As is well known, highorder CP calculations are difficult to perform, largely because the number of $\mathrm{CP}$ diagrams grows rapidly as one goes to higher orders in perturbation theory. The number of diagrams at third order is already quite large, though still manageable. Primarily because of this difficulty, a complete fourth-order calculation has never been carried out. It was soon realized that an order-by-order calculation of CP diagrams beyond third order is not practicable. To fully assess the effects of core polarization to high order, a non-perturbative method is called for.

In the present work, we shall use a non-perturbative method to carry out an all-order summation of CP diagrams. Our method is based on the elegant and rigorous induced interaction approach of Kirson [7] and Babu and Brown [8], hereafter referred to as the KBB method. In this formalism the vertex functions are obtained by solving a set of self-consistent equations, thereby generating $\mathrm{CP}$ diagrams to all orders in a manner similar to the parquet summation 9]. Using this approach, Kirson has studied ${ }^{18} \mathrm{O}$ and ${ }^{18} \mathrm{~F}$ using a $G$-matrix interaction, and Jopko and Sprung 10] have carried out a model study of this approach using a separable interaction. A main conclusion of both studies is that when $\mathrm{CP}$ diagrams are included to all orders the effective interaction is very close to that given by the bare interaction alone. In contrast, Sjöberg [11] applied the Babu-Brown formalism to nuclear matter and found that the inclusion of CP diagrams to all orders has a significant effect on the Fermi liquid parameters, in comparison with those given by the bare interaction. These conflicting results for $\mathrm{CP}$ studies of finite nuclei and infinite nuclear matter have served as a primary motivation for our present re-examination of the CP effect.

Our application of the KBB formalism to shell-model effective interactions is similar to that of Kirson, but our treatment is different in a number of important regards. As we will discuss, the particle-core and holecore coupling vertices used in the present work include a larger class of diagrams than have been previously studied. We will show how the inclusion of these diagrams is facilitated by using the recently developed low-momentum nucleon-nucleon interaction $V_{\text {low-k }}$ 12, 13, 14, 15, 16, 17, 18] instead of the previously used 

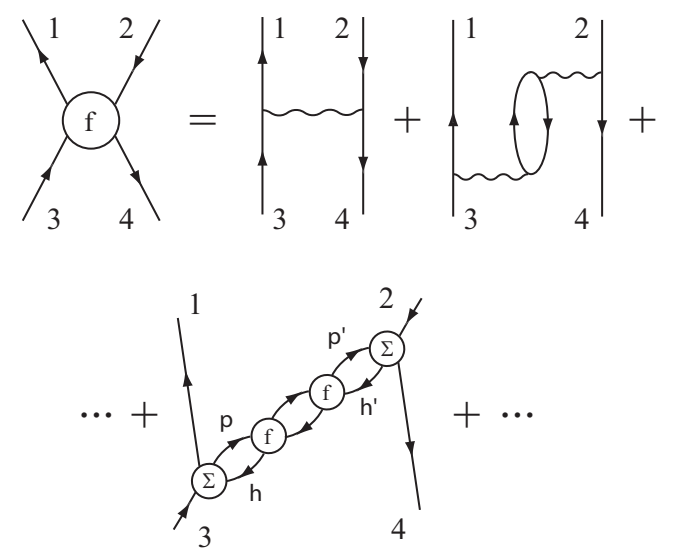

FIG. 1: Self-consistent diagramatic expansion of the $p h$ vertex function, $f$, where $\Sigma$ is defined in the text.

$G$-matrix. This is primarily because the $G$-matrix [6, 19] depends on both starting energy and Pauli exclusion operator, while $V_{\text {low-k }}$ depends on neither. It is noted that the S-wave interactions calculated from the MoszkowskiScott separation method gave essentially the same matrix elements as $V_{\text {low-k. }}$.20] In the subsequent Formalism section we will discuss these topics in greater detail, and in the final section we present our results together with a summary. Although the present calculation is restricted to the $s d$-shell effective interactions, the methods we develop can be readily expanded to other nuclear regions and with appropriate generalization applied to effective operators such as magnetic moments.

Formalism.- The KBB induced interaction approach provides a very appealing way for summing up planar diagrams to all orders. Its fundamental requirement is that the irreducible vertex functions be calculated selfconsistently. This means that any core polarization term contained in a vertex function must be generated selfconsistently from the same vertex function. To illustrate this point, we consider the particle-hole $(p h)$ vertex function $f$. As shown in Fig. 1. $f$ is generated by summation of the driving term $V$ and core polarization terms, the latter being dependent on $f$. This then gives the selfconsistent equation for $f$

$$
f=V+\Sigma g_{p h} \Sigma+\Sigma g_{p h} f g_{p h} \Sigma+\Sigma g_{p h} f g_{p h} f g_{p h} \Sigma+\cdots,
$$

where $g_{p h}$ is the free $p h$ Green function, and $\Sigma$ denotes the vertex for particle-core and hole-core coupling. The second-order CP diagram of Fig. 1 is the lowest-order term contained in $\Sigma g_{p h} \Sigma$. We note that for simplicity the bra and ket indices have been suppressed in the above equation as well as the equations that follow. For example, in Eq. (II) the $f$ on the LHS represents $\left\langle 12^{-1}|f| 34^{-1}\right\rangle$, while the fifth and sixth $\Sigma$ 's on the RHS represent $\left\langle 1 p h^{-1}|\Sigma| 3\right\rangle$ and $\left\langle 2^{-1}|\Sigma| p^{\prime} h^{\prime-1} 4^{-1}\right\rangle$, respectively. To further visualize the structure of $f$, let us consider $\Sigma=V$ in Eq. (1):

$$
f=V+V g_{p h} V+V g_{p h} f g_{p h} V+V g_{p h} f g_{p h} f g_{p h} V+\cdots \text {. }
$$

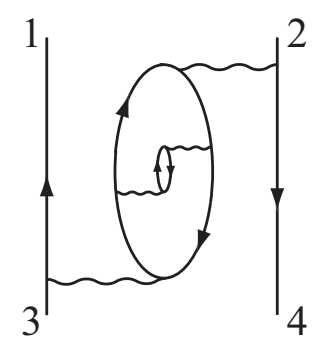

(a)

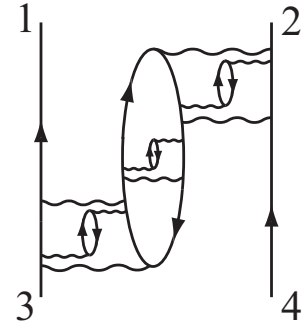

(b)
FIG. 2: Higher-order terms contributing to the vertex functions $f$ and $\Gamma$, including (a) nested bubbles in bubbles and (b) particle-core and hole-core couplings.

Since $f$ appears on both sides of this equation, it is clear that an iterative solution for $f$ will yield core polarization diagrams to all orders, including those with "bubbles inside bubbles", like those shown in diagram (a) of Fig. 2

For nuclear many-body calculations in general, we also need the particle-particle $(p p)$ vertex function $\Gamma$. Like $f, \Gamma$ is given by a driving term plus core polarization terms. Furthermore, the diagramatic representation of $\Gamma$ is identical to Fig. 1 except that the hole lines 2 and 4 are replaced by corresponding particle lines. This gives the self-consistent equation for $\Gamma$

$$
\Gamma=V+\Sigma g_{p h} \Sigma+\Sigma g_{p h} f g_{p h} \Sigma+\Sigma g_{p h} f g_{p h} f g_{p h} \Sigma+\cdots .
$$

To clarify our compact notation, we note that the external lines of the $\Sigma$ vertices in $\Gamma$ are different than those shown in Fig. 1 The upper $\Sigma$ vertex, for example, now represents $\left\langle 2|\Sigma| p h^{-1} 4\right\rangle$. These different $\Sigma$ vertices can be related to each other, however, via appropriate particlehole transformations.

Finally, the vertex functions $f$ and $\Gamma$ are coupled together via the coupling vertex $\Sigma$. In the present work we choose

$$
\begin{gathered}
\Sigma=V+\Sigma_{p h}+\Sigma_{p p} \\
\Sigma_{p h}=V g_{p h} V+V g_{p h} f g_{p h} V+V g_{p h} f g_{p h} f g_{p h} V+\cdots, \\
\Sigma_{p p}=V g_{p p} V+V g_{p p} \Gamma g_{p p} V+V g_{p p} \Gamma g_{p p} \Gamma g_{p p} V+\cdots,
\end{gathered}
$$

where $g_{p p}$ is the free $p p$ Green function.

The self-consistent vertex functions $f$ and $\Gamma$ are determined from Eqs. (11) and (3 6). These are similar to the equations used by Kirson [], except that our $\Sigma$ includes both $\Sigma_{p h}$ and $\Sigma_{p p}$, while the equivalent term in Kirson's calculations only includes $\Sigma_{p h}$ [7, 21]. To see the role of the $\Sigma$ vertices, let us consider diagram (b) of Fig. 2. Here the lower particle-core vertex, which contains repeated particle-particle interactions, belongs to $\Sigma_{p p}$, while the upper one, which contains repeated particle-hole interactions, belongs to $\Sigma_{p h}$. It is, of course, necessary to include $\Sigma_{p p}$ in order to have such CP diagrams in the all-order sum. Our equations are equivalent to those of Kirson when $\Sigma_{p p}$ is set to zero, and in this case $\Gamma$ does not enter the calculation of $f$. 
Solving the above equations for $f$ and $\Gamma$ may seem complicated, but we have found their solution can be simplified significantly through use of the true $p h$ and $p p$ Green functions

$$
\begin{gathered}
G_{p h}=g_{p h}+g_{p h} f G_{p h}, \\
G_{p p}=g_{p p}+g_{p p} \Gamma G_{p p} .
\end{gathered}
$$

Using these Green functions to partially sum and regroup our series, the self-consistent Eqs. (11) and (3, 6) assume a much simpler form

$$
\begin{gathered}
f=V+\Sigma G_{p h} \Sigma, \\
\Gamma=V+\Sigma G_{p h} \Sigma, \\
\Sigma=V+V G_{p h} V+V G_{p p} V .
\end{gathered}
$$

The above simplifications also aid our numerical efforts, and using the following iterative method we find our coupled equations can be solved rather efficiently. For the $n^{\text {th }}$ iteration, we start from $f^{(n)}$ and $\Gamma^{(n)}$, to first calculate $G_{p h}^{(n)}$ and $G_{p p}^{(n)}$ followed by $\Sigma^{(n)}$, as seen from Eqs. (7111). The vertex functions for the subsequent iteration are then obtained by taking $f^{(n+1)}=V+$ $\Sigma^{(n)} G_{p h}^{(n)} \Sigma^{(n)}$ and $\Gamma^{(n+1)}=V+\Sigma^{(n)} G_{p h}^{(n)} \Sigma^{(n)}$. The entire iterative process begins from the initial $f^{(0)}=V+V g_{p h} V$ and $\Gamma^{(0)}=V+V g_{p h} V$, and typically converges after just a few iterations.

In the present work, we have included folded diagrams to all orders. As detailed in 22], we use this method to reduce the full-space nuclear many-body problem $H \Psi_{n}=$ $E_{n} \Psi_{n}$ to a model space problem $H_{\text {eff }} \chi_{m}=E_{m} \chi_{m}$, where $H=H_{0}+V, H_{\text {eff }}=H_{0}+V_{\text {eff }}$ and $V$ denotes the bare $\mathrm{NN}$ interaction. The effective interaction $V_{\text {eff }}$ is given by the folded-diagram expansion

$$
V_{\mathrm{eff}}=\hat{Q}-\hat{Q}^{\prime} \int \hat{Q}+\hat{Q}^{\prime} \int \hat{Q} \int \hat{Q}-\cdots .
$$

We consider the effective interactions for valence nucleons in the $s d$-shell, and in this case $\hat{Q}$ is given by the vertex function $\Gamma$ obtained earlier from the KBB equations. In 7], the effect of higher order CP diagrams to the nonfolded $\hat{Q}$ term was extensively studied. In the present work, we first calculate $\hat{Q}$ including CP diagrams to all orders. Then the above folded diagram series for $V_{\text {eff }}$ is summed to all orders using the Lee-Suzuki iteration method [6], explicitly including folded CP diagrams to all orders.

For the present calculation we have chosen to use the low-momentum nucleon-nucleon interaction, $V_{\text {low-k }}$. Since the vertex functions $f$ and $\Gamma$ both depend on the starting energy, there would be off-energy-shell effects present in many $\mathrm{CP}$ diagrams if the $G$-matrix interaction were chosen. This would make the calculation very complicated. $V_{\text {low-k }}$, on the other hand, is energy independent so no such difficulties are encountered. Since

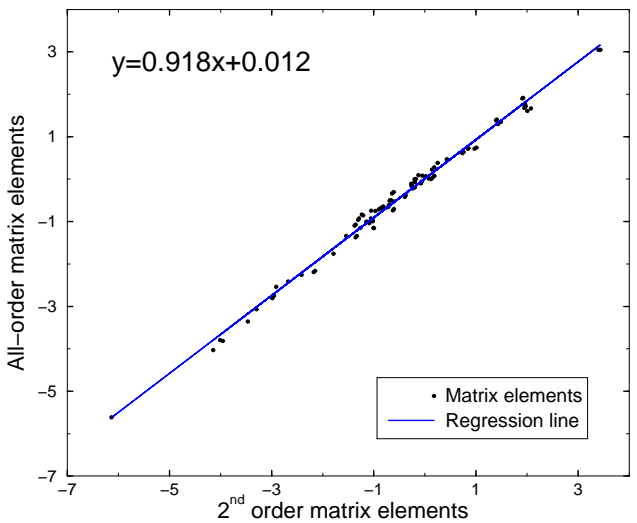

FIG. 3: A comparison of the second-order core polarization matrix elements with those of the all-order KBB calculation.

detailed treatments of $V_{\text {low-k }}$ have been given elsewhere $12,13,14,15,16,17,18$, , here we provide only a brief description. We define $V_{\mathrm{low}-\mathrm{k}}$ through the $T$-matrix equivalence equations

$$
\begin{gathered}
T\left(k^{\prime}, k, k^{2}\right)=V_{N N}\left(k^{\prime}, k\right)+P \int_{0}^{\infty} q^{2} d q V_{N N}\left(k^{\prime}, q\right) \\
\times \frac{1}{k^{2}-q^{2}} T\left(q, k, k^{2}\right) \\
T_{l o w-k}\left(p^{\prime}, p, p^{2}\right)=V_{l o w-k}\left(p^{\prime}, p\right) \\
+P \int_{0}^{\Lambda} q^{2} d q V_{l o w-k}\left(p^{\prime}, q\right) \frac{1}{p^{2}-q^{2}} T_{l o w-k}\left(q, p, p^{2}\right) \\
T\left(p^{\prime}, p, p^{2}\right)=T_{l o w-k}\left(p^{\prime}, p, p^{2}\right) ;\left(p^{\prime}, p\right) \leq \Lambda,
\end{gathered}
$$

where $V_{N N}$ represents some realistic $\mathrm{NN}$ potential and $\Lambda$ is the decimation momentum beyond which the high-momentum components of $V_{N N}$ are integrated out. $V_{\text {low-k }}$ preserves both the deuteron binding energy and the low-energy scattering phase shifts of $V_{N N}$. Since empirical nucleon scattering phase shifts are available only up to the pion production threshold $\left(E_{l a b} \sim 350 \mathrm{MeV}\right)$, beyond this momentum the realistic NN potentials cannot be uniquely determined. Accordingly, we choose $\Lambda \approx 2.0 \mathrm{fm}^{-1}$ thereby retaining only the information from a given potential that is constrained by experiment. In fact for this $\Lambda$, the $V_{\text {low-k }}$ derived from various $\mathrm{NN}$ potentials [23, 24, 25, 26] are all nearly identical [16]. Except where noted otherwise, in our calculations we employ the $V_{\text {low-k }}$ derived from the CD-Bonn potential 23]. Results and Discussion.- As an initial study, we have carried out a restricted all-order CP calculation for the $s d$-shell effective interactions. In particular, we sum only the TDA diagrams for the Green functions $G_{p p}$ and $G_{p h}$, leaving a study of RPA diagrams to a future publication. Also, we use a limited shell model space consisting of 10 orbits from $0 s_{1 / 2}$ to $1 p_{1 / 2}$ and an oscillator constant of $\hbar \omega=14 \mathrm{MeV}$. Only core excitations within this space are included. Vary, Sauer and Wong 27] have pointed out 

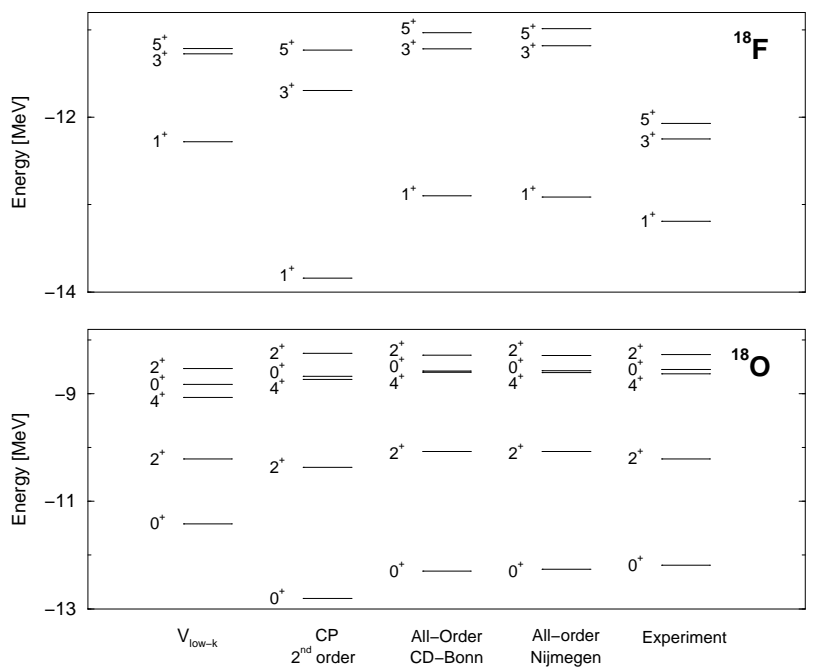

FIG. 4: Spectra for the ${ }^{18} \mathrm{~F}$ (top) and ${ }^{18} \mathrm{O}$ (bottom) systems calculated to different orders in perturbation theory.

that for CP diagrams one needs to include intermediate states of high excitation energies (up to $\sim 10 \hbar \omega$ ) in order for the second-order core polarization term to converge. In their work a $G$-matrix derived from the Reid softcore potential was used, but our use of $V_{\text {low-k }}$ may yield different results as it has greatly reduced high-momentum components. We plan to study this convergence problem for $V_{\text {low-k }}$ in the near future.

With these restrictions, we have calculated $V_{\text {eff }}$ from Eqs. (77 12). A large number of angular momentum recouplings are involved in calculating the $\mathrm{CP}$ diagrams. In this regard, we have followed closely the diagram rules in 28]. In Fig. 3] we compare the $s d$-shell $V_{\text {eff }}$ matrix elements calculated with all-order CP diagrams with those from second-order 12 CP diagrams. A least-squares fit was applied to the data, and it is apparent that the effect of including CP to all orders in our calculation is a mild suppression of the second-order contributions. This conclusion is further born out in the calculation of the ${ }^{18} \mathrm{O}$ and ${ }^{18} \mathrm{~F}$ spectra, the results of which are shown in Fig. [4 Here we observe a weak suppression of the second-order effects in ${ }^{18} \mathrm{O}$ but a moderate suppression in ${ }^{18} \mathrm{~F}$. In the same figure we also observe that the spectra for different $V_{\text {low-k }}$ derived from the CD-Bonn and Nijmegen bare potentials are nearly identical.

In summary, we have presented a method based on the KBB induced interaction formalism for efficiently summing core polarization diagrams to all orders in perturbation theory. This summation is carried out by way of the KBB self-consistent equations whose solution is significantly simplified by the use of the true $p p$ and $p h$ Green functions, and by the use of the energyindependent $V_{\text {low-k }}$. Although our calculation was restricted in several important aspects, we find that our final renormalized interaction is remarkably close to that of second-order perturbation theory. This is of practical importance and a welcoming result, for it allows one to use the results from a second-order calculation to approximate the contributions resulting from a large class of higher-order diagrams. In the future we intend both to expand our treatment by including additional diagramatic contributions (RPA) and to use the results in calculations of other nuclear observables.

We thank M. Kirson for helpful discussions. Partial support from the US Department of Energy under contracts DE-FG02-88ER40388 is gratefully acknowledged.

[1] G. F. Bertsch, Nucl. Phys. 74, 234 (1965).

[2] T. T. S. Kuo and G. E. Brown, Nucl. Phys. 85, 40 (1966).

[3] G. E. Brown, Unified Theory of Nuclear Models and Forces (North-Holland, Amsterdam, 1971).

[4] A. P. Zuker, Phys. Rev. Lett. 90, 042502 (2003).

[5] B. R. Barrett and M. W. Kirson, Nucl. Phys. A148, 145 (1970).

[6] M. Hjorth-Jensen, T. T. S. Kuo, and E. Osnes, Phys. Rep. 261, 126 (1995), and references therein.

[7] M. W. Kirson, Ann. Phys. 66, 624 (1971); ibid. 68, 556 (1971); ibid. 82, 345 (1974).

[8] S. Babu and G. E. Brown, Ann. Phys. 78, 1 (1973).

[9] A. Jackson, A. Lande, and R. A. Smith, Phys. Rep. 86, 55 (1982); A. Lande and R. A. Smith, Phys. Rev. A 45, 913 (1992).

[10] D. W. L. Sprung and A. M. Jopko, Can. J. Phys. 50, 2768 (1972).

[11] O. Sjöberg. Ann. Phys. 78, 39 (1973).

[12] S. K. Bogner, T. T. S. Kuo, L. Coraggio, A. Covello, and N. Itaco, Phys. Rev. C 65, 051301(R) (2002).

[13] T. T. S. Kuo, S. K. Bogner, and L. Coraggio, Nucl. Phys. A704, 107c (2002).

[14] L. Coraggio et al., Phys. Rev. C 66, 021303(R) (2002).

[15] L. Coraggio, A. Covello, A. Gargano, N. Itaco, and T. T. S. Kuo, Phys. Rev. C 66, 064311 (2002).

[16] S. K. Bogner, T. T. S. Kuo, and A. Schwenk, Phys. Rep. 386, 1 (2003).

[17] J. D. Holt et al., Nucl. Phys. A733, 153 (2004).

[18] J. D. Holt, T. T. S. Kuo, and G. E. Brown, Phys. Rev. C 69, 034329 (2004).

[19] E. M. Krenciglowa et al., Ann. Phys. 101, 154 (1976).

[20] J. W. Holt and G. E. Brown, nucl-th/0408047

[21] P. J. Ellis and E. Osnes, Rev. Mod. Phys. 49, 777 (1977).

$[22]$ T. T. S. Kuo and E. Osnes, Lecture Notes in Physics (Springer-Verlag, New York, 1990), Vol. 364.

[23] R. Machleidt, Phys. Rev. C 63, 024001 (2001).

[24] R. B. Wiringa et al., Phys. Rev. C 51, 38 (1995).

[25] V. G. J. Stoks et al., Phys. Rev. C 49, 2950 (1994).

[26] D. R. Entem et al., Phys. Rev. C 65, 064005 (2002).

[27] J. P. Vary, P. U. Sauer, and C. W. Wong, Phys. Rev. C 7, 1776 (1973).

[28] T. T. S. Kuo et al., Ann. Phys. 132, 237 (1981). 